\title{
Pre-solar grains: outlook and opportunities for astrophysics
}

\author{
Larry R. Nittler and Conel M. O'D. Alexander \\ Department of Terrestrial Magnetism, Carnegie Institution of Washington, 5241 Broad Branch \\ Road NW, Washington, D.C. 20015, USA \\ email: alexande,lrn@dtm.ciw.edu
}

\begin{abstract}
As pristine condensates from a large number of stars, pre-solar grains provide unique information on the sources, types, compositions and processing histories of dust in the Galaxy. However, their promise remains largely unfulfilled. Here we discuss some of the astrophysical problems for which pre-solar grains might provide important insights and constraints in coming years.
\end{abstract}

Keywords. dust, extinction, circumstellar matter, stars: AGB and post-AGB, supernovae, nuclear reactions, nucleosynthesis, abundances

\section{Introduction}

Pre-solar grains in meteorites and interplanetary dust particles (IDPs) are relatively pristine samples of circumstellar dust that can be studied in remarkable detail by advanced microanalytical tools in the laboratory (e.g., Nittler 2003). The types of grains that have been found include silicates, oxides, graphite, diamond, $\mathrm{SiC}$ and $\mathrm{Si}_{3} \mathrm{~N}_{4}$. They appear to come from red giant branch (RGB) and asymptotic giant branch (AGB) stars, from supernovae and possibly novae. Their analysis can provide elemental and isotopic compositions that are far more precise than is possible astronomically, as well as powerful new information that cannot be obtained astronomically but which complements that obtained from traditional astronomical observations. Many examples are discussed in the other papers from this Joint Discussion. However, the promise of pre-solar grains for astronomy and astrophysics has not been fulfilled to the extent it could be. With several notable exceptions, relatively few studies by traditional astronomers and astrophysicists have taken into account the growing observational data set on pre-solar grains. This is in part due to the fact that for much of the nearly twenty years since pre-solar grains were discovered, most of the studied grains have been atypical, from the astronomical point of view, for circumstellar and interstellar dust. For example, until recently, only grains larger than about $\sim 1 \mu \mathrm{m}$ in diameter were amenable to isotopic analysis as single grains, while typical circumstellar and interstellar dust sizes are $\sim 100 \mathrm{~nm}$. Similarly, silicates are the dominant type of dust in the Galaxy, but these have been identified in the pre-solar grain population only quite recently (Messenger et al. 2003). The situation has greatly improved with recent technological advances. For example, isotopic measurements can now be made on individual grains down to $100 \mathrm{~nm}$ in size, and identification of pre-solar silicate grains for detailed study has become fairly routine. Here we discuss some of the astrophysical problems for which pre-solar grains might provide important insights and constraints in coming years. 


\section{Opportunities for astrophysics}

\subsection{Galactic chemical evolution $(G C E)$}

It is now well-established that the isotopic compositions of some elements, notably Si, $\mathrm{Ti}$, and $\mathrm{O}$, in many pre-solar grains reflect GCE processes (Clayton \& Timmes 1997; Alexander \& Nittler 1999), but a unified quantitative explanation of the data in terms of GCE is lacking. Unfortunately, at present the limitations of both the grain data set and GCE models make it difficult to judge the relative merits of the disparate ideas put forward to explain the grain data. Perhaps the best hope for progress from the grain front is the acquisition of multi-element data for a large number of grains of different types, for example $\mathrm{Si}$, Ti and $\mathrm{O}$ isotopic data for pre-solar silicates, $\mathrm{SiC}$ and oxide phases from AGB stars. Such a multi-dimensional dataset would allow the disentanglement of GCE processes from nuclear processes in the parent stars of the grains and could serve as high-precision constraints on future GCE models.

\subsection{Stellar evolution and nucleosynthesis}

Observational data from both stars and pre-solar grains clearly demonstrate the existence of an 'extra mixing' process (often called 'cool bottom processing' or CBP, Wasserburg et al. 1995) occurring in low-mass RGB and AGB stars. What is still unknown is the physical-dynamical cause of CBP. In addition to indicating the existence of CBP, presolar grains may also shed light on the origin and nature of the mixing. First, comparison of grain data with parameterized models (e.g., Nollett et al. 2003) provides constraints on the physical conditions of the mixing (e.g., mixing rate, temperature). Second, the distribution of mixing parameters indicated by the isotopic data, including the lack of mixing in many parent stars, must be reproduced by any reasonable model of CBP.

One of the biggest mysteries of pre-solar grain studies is the origin of the highly ${ }^{13} \mathrm{C}$ enriched SiC grains, known as Types A and B (Amari et al. 2001). J-type and CH-type $\mathrm{C}$-stars are known to be ${ }^{13} \mathrm{C}$-rich $\mathrm{C}$ stars, although their origins are also mysterious. The ratio of $\mathrm{A}+\mathrm{B}$ grains to normal $\mathrm{AGB}$ grains is very similar to the ratio of J-type and N-type C-stars. Thus, it seems likely that most of the A+B grains come from J-type stars. Whether from J-type or CH-type stars, the detailed information that will come from the $\mathrm{A}+\mathrm{B}$ grains should greatly enhance our knowledge of how these stars form and evolve.

A fraction of pre-solar grains originated in supernova explosions and their compositions provide detailed information about nucleosynthesis and mixing processes in such environments (see e.g., Amari \& Lodders, this volume). This topic is likely to expand in coming years with more detailed grain data, with detailed observations of supernova remnants using the Chandra and Spitzer telescopes, and with multi-dimensional modeling of supernovae, now becoming possible with modern computers.

\subsection{Dust formation around stars}

How dust grains nucleate and grow in stellar environments is not well understood, despite being central to models of dust production in the Galaxy, the evolution of dust in the ISM, and mass loss from stars. Pre-solar grains hold great promise for improving understanding in this field, since formation processes are recorded in the detailed structures and compositions of the grains that can be characterized in minute detail. Thermodynamic equilibrium calculations have been very useful for determining the types of grains that should form and the order in which they condense (Lodders \& Fegley 1995). However, astronomical and astrophysical evidence shows that thermodynamic equilibrium is not maintained in stellar outflows. A realistic picture of grain formation will require kinetic 
models of grain nucleation and growth, coupled to physical models of the conditions that exist in stellar outflows of different kinds (e.g., Gail \& Sedlmayr 1999)

Pre-solar grains will provide a wealth of constraints for these models. The size distribution of grains reflects their growth conditions, but there seems to be only modest variations in the range of pre-solar grain compositions with size. So, for instance, different types (masses/metallicities) of AGB stars produce SiC grains with similar size distributions, as do supernovae. Perhaps the similarities in the size distributions of grains from these different objects reflects some common underlying mechanism.

Formation of grains that range in size from $100 \mathrm{~nm}$ or less to several microns seems to require a range of gas densities in outflows. In particular, formation of large $(>1 \mu \mathrm{m})$ AGB grains on reasonable timescales requires much higher densities than estimated average densities in a uniform radially symmetric outflow (e.g., Bernatowicz et al. 2005). The density variations recorded by the grains are probably caused by shocks in the outflows that are observed astronomically, and highlight the need for two- and three-dimensional models of grain growth in outflows.

The microstructures of grains also provide clues to the mechanisms of nucleation and growth. Heterogeneous nucleation on pre-existing grains is often invoked to overcome the kinetic barriers to spontaneous nucleation in the gas phase. However, of the grain types that have been examined, only certain types of graphite grain show clear evidence for heterogeneous nucleation (Bernatowicz et al. 1996). For thermodynamic and/or kinetic reasons, minerals with the same compositions can have different crystal structure (polytype), depending on the conditions under which they form. Whether $\mathrm{AGB} \mathrm{Al}_{2} \mathrm{O}_{3}$ grains are crystalline or amorphous may depend on the availability of $\mathrm{Ti}$ when they grow (Stroud et al. 2006). SiC has many polytypes whose stability and preservation depends on the temperature of formation and the rate at which the system cools (Daulton et al. 2002).

Dust formation in supernovae ( $\mathrm{SNe}$ ) is of great current interest in astrophysics, both because of observations of copious dust at high redshifts (Bertoldi et al. 2003) and because of observations of dust in Galactic SN remnants (Sugerman et al. 2006). However, the processes of dust formation in SNe and the types of dust produced are poorly understood. Grains from supernovae have been found in almost every pre-solar grain type and again they provide an opportunity for making progress in this field. For example, in contrast to pre-solar grains from AGB stars, which are typically single euhedral crystals, the $\mathrm{SNe}$-derived $\mathrm{SiC}$, silicates and $\mathrm{Si}_{3} \mathrm{~N}_{4}$ grains studied to date by transmission electron microscopy are polycrystalline aggregates of smaller 10-100 nm sized sub-grains (Stroud et al. 2004; Messenger et al. 2005; Stroud et al. 2006), indicating different conditions during grain formation in the different environments. Micron- to ten micron-sized graphitic grains from supernovae contain sub-crystals of $\mathrm{Fe}-\mathrm{Ni}$ metal and $\mathrm{TiC}$, providing tight constraints on the conditions where the grains formed (Lodders 2006). Isotopic evidence from $\mathrm{SiC}$ and diamond grains also provide clues to the timing of grain condensation in supernovae ejecta.

\subsection{Sources and processing of interstellar dust}

Pre-solar grains come from at least tens and, perhaps, hundreds of stars (Alexander 1997) with a considerable range in masses and metallicities. To date, there has been no quantitative explanation for how grains from so many stars became part of the presolar molecular cloud. Based on observed encounter rates, direct injection of dust from stars passing through a cloud cannot be the explanation. Presumably, the grains are accumulated by a molecular cloud as it forms, and the number of stellar sources for grains must in some way reflect the volume of the Galaxy sampled by the forming cloud. 
Circumstellar grains are thought to be rapidly processed in the ISM by supernovadriven shock waves (Slavin et al. 2004). This processing results in the rapid amorphisation of crystalline silicates, sputtering and cratering/shattering in grain-grain collisions. The pre-solar grains appear to be relatively unprocessed, which is surprising given the rapidity of the ISM processing and the number of stars represented. Obviously, a small fraction of circumstellar grains are able to survive in the ISM and those that do survive appear to be largely unscathed. How this might happen in the context of current models of dust evolution in the ISM has yet to be explored.

Given that pre-solar grains come from so many sources and seem to be relatively unprocessed, they also provide constraints on circumstellar dust production rates in the Galaxy. Astronomical estimates of Galactic dust production rates vary widely, particularly in the amount of dust produced by supernovae. The relative abundances of different types of pre-solar grain from various stellar sources are generally roughly what is expected from astronomical estimates of stellar dust production rates that only require a few percent of the dust coming from supernovae (Alexander 1997). The two glaring differences are the low abundances of graphite grains and high abundance of nanodiamonds amongst circumstellar grains in meteorites.

\section{References}

Alexander, C. M. O'D. 1997, in: T. J. Bernatowicz \& E. K. Zinner (eds.), Astrophysical Implications of the Laboratory Study of Pre-Solar Materials, AIP-CP, 402, 567

Alexander, C. M. O'D., \& Nittler, L. R. 1999, ApJ, 519, 222

Amari, S., Nittler, L. R., Zinner, E., Lodders, K., \& Lewis, R. S. 2001, ApJ, 559, 463

Bernatowicz, T. J., Cowsik, R., Gibbons, P. C., Lodders, K., Fegley, B., Amari, S., \& Lewis, R. S. 1996, ApJ, 472, 760

Bernatowicz, T. J., Akande, O. W., Croat, T. K., \& Cowsik, R. 2005, ApJ, 631, 988

Bertoldi, F., Carilli, C. L., Cox, P., Fan, X., Strauss, M. A., Beelen, A., Omont, A., \& Zylka, R. 2003, A\&A (Letters), 406, L55

Clayton, D. D., \& Timmes, F. X. 1997, in: T. J. Bernatowicz \& E. K. Zinner (eds.), Astrophysical Implications of the Laboratory Study of Pre-Solar Materials, AIP-CP, 402, 237

Daulton, T. L., Bernatowicz, T. J., Lewis, R. S., Messenger, S., Stadermann, F. J., \& Amari, S. 2002, Science, 296, 1852

Gail, H.-P., \& Sedlmayr, E. 1999, A\&A, 347, 594

Lodders, K., \& Fegley, B. 1995, Meteoritics, 30, 661

Lodders, K. 2006, ApJ (Letters), 647, L37

Messenger, S., Keller, L. P., \& Lauretta, D. S. 2005, Science, 309, 737

Messenger, S., Keller, L. P., Stadermann, F. J., Walker, R. M., \& Zinner, E. 2003, Science, 300, 105

Nittler, L. R. 2003, Earth Planet. Sci. Lett., 209, 259

Nollett, K. M., Busso, M., \& Wasserburg, G. J. 2003, ApJ, 582, 1036

Slavin, J. D., Jones, A. P., \& Tielens, A. G. G. M. 2004, ApJ 614, 796

Stroud, R. M., Nittler, L. R., \& Alexander, C. M. O'D. 2004, Science 305, 1455

Stroud, R. M., Nittler, L. R., \& Alexander, C. M. O'D. 2006, Meteorit. Planet. Sci. Supp., 41 (Supp.), Abstract \#5360

Stroud, R. M., Nittler, L. R., \& Hoppe, P. 2004, Meteorit. Planet. Sci., 39 (Supp.), Abstract \#5039

Sugerman, B. E. K., Ercolano, B., Barlow, M. J., et al. 2006, Science, 313, 196

Wasserburg, G. J., Boothroyd, A. I., \& Sackmann, I.-J. 1995, ApJ (Letters), 447, L37 